\title{
Desigualdad en la calidad de la educación en el Brasil: un estudio de caso en la región noroeste fluminense
}

\author{
Marlon Gomes Ney \\ Marlúcia Degli Esposti Tiradentes \\ María Eugênia Ferreira Totti
}

\begin{abstract}
Brasil, like many countries in Latin America, has a strong inequality in income distribution and opportunities among its population. In the educational realm, the XXth century saw the democratization of the access to education in this country. However, the expansion of the public educational network occurred without a warrant of quality for everyone and, therefore, the distinction between rich and poor survived in the educational system. This article presents the results of an investigation that, utilizes data from the national exam of secondary education to show the strong correlation between the socioeconomic conditions of the families and the quality of the basic education that can be enjoyed by the students of the province of Rio de Janeiro.
\end{abstract}

\section{Introducción}

El Brasil es un país campeón en desigualdad de renta. De acuerdo con un estudio del Instituto de Investigación Económica Aplicada de Brasil (IPEA 2005), la desigualdad de renta en el país es tan elevada que el 1\% más rico de la población se apropia del $13,0 \%$ de la renta total, valor ligeramente inferior a la proporción del 13,3 \% de la renta total apropiada por el 50\% más pobre. El índice Gini que mide la desigualdad en la distribución en la renta domiciliar per cápita es de 0,60, uno de los valores más altos del mundo. La concentración de la renta en el Brasil es uno los temas más discutidos en la economía, lo que puede ser comprobado por el gran número de estudios que procuran no solo acompañar su variación a lo largo del tiempo, sino también estimar los efectos 
de sus principales determinantes. En la literatura son comúnmente observados $\mathrm{y}$ analizados algunos factores estructurales que han contribuido para mantener las disparidades de rendimiento siempre en niveles muy elevados en el país: las desigualdades educacionales, la concentración de la propiedad de la tierra, los contrastes regionales, las desigualdades intersectoriales y la discriminación por sexo y por color.

El objetivo de este trabajo es analizar el efecto de la educación en las disparidades de renta en el país, destacando el papel que la calidad de la enseñanza básica tiene en la reproducción de altos niveles desigualdad. Por medio de un estudio de caso realizado en la región noroeste del estado de Rio de Janeiro, mostraremos que el proceso de democratización del acceso a la escuela pública ocurrió sin un gran compromiso con la calidad de la educación. El resultado es que el dualismo en el ambiente educacional brasilero es preservado, sólo que ahora cada vez menos por la exclusión de la población pobre de las escuelas y cada vez más de las buenas escuelas, siendo progresivamente instaurado por la oposición enseñanza pública versus privada.

El estudio está dividido en tres secciones. En la primera se hace un análisis histórico del desarrollo de la educación pública en el Brasil hasta ella tornarse una realidad de la gran mayoría de ciudadanos. Así, se muestran las raíces y características de la existencia del dualismo escolar brasilero, hoy fuertemente marcado por las diferencias en la calidad de la educación básica. La segunda sección asocia la alta concentración de renta en el Brasil con las desigualdades de oportunidades educacionales, discutiendo no sólo el sentido de causalidad educación-renta, sino también su relación en sentido inverso: renta-educación. Finalmente, en la tercera sección, es realizado un estudio de caso sobre la desigualdad en la calidad de la educación en el Noroeste Fluminense, la más pobre de las mesoregiones del estado de Rio de Janeiro.

\section{La democratización del acceso a la educación básica en el Brasil}

El Brasil entra para la historia de la llamada civilización Occidental exactamente al abrirse el siglo XVI. Su historia coincide, con el periodo caracterizado por el surgimiento de la educación pública (Saviani, 1997: 4).

Nuestra educación básica nace bajo la forma de educación pública religiosa con los jesuitas. A pesar de estar bajo los criterios de la Iglesia católica, puede ser considerada pública por haber recibido incentivo y subsidio de la carona portuguesa, una vez que era el Estado portugués quien la financiaba. Su inicio 
se da en el siglo XVI y se extiende hasta mediados del siglo XVIII. La educación jesuita tiene su fin en la era de Pombal, quien al expulsar a los jesuitas del Brasil posibilita, según Saviani (2005), un modelo para una escuela pública estatal.

Las reformas pombalinas se contraponen al predominio de las ideas religiosas y, con base en las ideas laicas inspiradas en el iluminismo, instituyen el privilegio del Estado en materia de instrucción surgiendo así nuestra versión de la educación pública estatal (Saviani, 2005: 9).

Con la aprobación de la ley de las escuelas de primeras letras de 1827, cerca de cinco años después de la independencia del Brasil y de la instauración del Imperio, el país casi creó su primer modelo de escuela pública nacional. Según Saviani (2005), sin embargo el Acto Adicional de 1834 colocó las escuelas primarias bajo responsabilidad de las provincias, hecho que inviabilizó la existencia de esta consciencia nacional. Con una mala organización, principalmente en lo que se refiere a la recaudación tributaria, la escuela, sobre todo la secundaria, quedó bajo la responsabilidad de la iniciativa privada, hecho que acentuó el carácter elitista de la educación (Romanelli, 2005).

La voluntad de tornar la educación brasilera universal y gratuita surge con los ideales republicanos democráticos a finales del siglo XIX. A partir de ese momento "el poder público asume la tarea de organizar y mantener integralmente las escuelas teniendo como objetivo la difusión de la enseñanza a toda la población" (Saviani, 2005: 10). La organización social en el Brasil se modifica, ocasionando el surgimiento de una camada intermediaria que ve en la educación una forma de ascensión social.

Desde mucho antes, el título de doctor valía tanto como el de propietario de tierras, con garantía para la conquista de prestigio social y de poder político. Era comprensible, por tanto, que, desprovista de tierras fuese para el título que esa pequeña burguesía apelara, con el fin de afirmarse como clase y asegurarse el estatus al cual aspiraba (Romanelli, 2005: 37).

Romanelli (2005) presenta una cuestión importante, la visión brasilera sobre el trabajo físico como algo degradante, herencia de la esclavitud; de allí la consideración del ocio como distinción de clase. Como también afirma Teixeira (1994), la escuela brasilera "clasifica" sus alumnos. Pasar por ella significa especializarse para la clase media o superior: "Ser educado escolarmente significa, en el Brasil, no ser operario, no ser miembro de las clases trabajadoras" (Teixeira, 1994: 50). En la visión de Teixeira (1994), esa revolución democrática, con el surgimiento de la clase media, hace que nuestra escuela siga el modelo europeo con educación dualista, con escuelas para el pueblo y para la elite. La educación 
pública común a todos es condición necesaria para la democratización del acceso a la escuela, teniendo en cuenta que casi exclusivamente la elite tiene condición de asumir los gastos de la escuela privada. Ideológicamente correcta, la república vieja no fue capaz de realizar sus ideales, y la estructura dualista de la educación brasilera se mantuvo.

A pesar de existir la intención de difundir la educación, desde las primeras escuelas creadas por el Imperio brasilero, existía un alto criterio de selección que puede ser visualizado en el ejemplo que Nosella (1997) presenta:

El examen de suficiencia para el ingreso en la Escuela Normal Secundaria de San Carlos, en enero de 1931, comprueba ese rigor en los estudios. Participaba del comité examinador el educador Lourenço Filho. Se presentaron 119 candidatos. En la primera fase (aritmética) fueron aprobados apenas ocho (8). Al final pasaron solamente cuatro (4) (Nosella, 1997: 170).

Es evidente que la rigidez no siempre alcanzaba estos niveles. No obstante es importante dejar claro que la idea de universalizar la educación no era vista como sinónimo de desvalorización de su calidad.

Obviamente, en una sociedad fuertemente dualista, cualquier rigor en los estudios terminaría por "excluir" de la escuela un inmenso número de ciudadanos. La verdad sea dicha, la causa principal de esta exclusión no podría ser atribuida al rigor escolar y sí a la propia sociedad dicotómica, a la República socialmente dual (Nosella, 1997: 171).

Se hace claro que la intención del Estado no era populista, una vez que el sistema de enseńanza acababa por excluir de la escuela a las masas populares, principalmente en el caso de las Escuelas Normales que tenían por clientela a las hijas de los hacendados, de los altos funcionarios públicos y grandes negociantes, atendiendo a los intereses de las clases dominantes de la primera república (Nosella, 1997).

Con el inicio del proceso de urbanización e industrialización, las transformaciones tecnológicas y sociales derivadas de él tornan a la cultura letrada todavía más necesaria. Diferente de la vida en el campo, donde las técnicas de producción agrícola son más rudimentarias y una parte significativa de la producción es utilizada para el autoconsumo, en las ciudades gran parte de lo que se produce es comercializado y las técnicas de producción y el ambiente fabril exigen mayor nivel de instrucción:

En este proceso, el campo es subordinado a la ciudad y la agricultura a la industria que realiza la conversión de la ciencia, potencia espiritual, en potencia material. El predominio de la ciudad y de la industria 
sobre el campo y la agricultura tiende a generalizarse, y a ese proceso corresponde la exigencia de la generalización de la escuela. Así, no es por coincidencia que la constitución de la sociedad burguesa trajera consigo la bandera de la escolarización universal y obligatoria (Saviani, 1997: 2).

Aliado a estas transformaciones surge el populismo, en la era Vargas (19301954), que trata de administrar los conflictos sociales propios del proceso de industrialización y modernización de la economía, buscando preservar el poder de la elite agraria brasilera. Transmitiendo una falsa idea de unidad, su objetivo es apaciguar conflictos oriundos de las grandes diferencias regionales y sociales del país. Es importante observar esto para entender las transformaciones de la educación escolar que suceden en este periodo. Según el mismo autor, el populismo atribuye el fracaso de la democratización de la educación al alto rigor que la escuela elitista imponía. Para solucionar el problema, desfigura el método de enseñanza y rebaja la calidad:

Esto es, crea una escuela aparente. En esta época, el mundo del trabajo se aproxima al mundo de la escuela, pero los dos mundo no se integran; los dirigentes y los dirigidos continúan en escuelas diferentes así sea recibiendo diplomas "iguales". El idealismo populista que piensa en crear la escuela "unitaria”, abre al trabajador un largo camino de una escuela secundaria empobrecida sin proporcionarle medios para recorrer los años de la universidad. Es un ideal cínico (Nosella, 1997: 175).

Teixeira (1994) es un autor que se suma a la crítica de Nosella (1997), ya que también censura la falta de cuidado con que fue hecha la expansión de la enseñanza en el Brasil. Él destaca la creación de las "escuelas-cine", término idealizado por el autor debido al surgimiento de tres y hasta cuatro turnos por día en la escuela como si fuesen sesiones de cine, con la intención de obtener y atender un número cada vez mayor de alumnos. Según Nosella (1997), se dio además el rápido crecimiento de la enseñanza nocturna; no obstante, ella en su mayoría carecía de calidad, convirtiendo el diploma en la principal motivación para la proliferación de cursos.

La falta de cuidado con las escuelas ya existentes es un triste factor para tener en cuenta, pues el objetivo de expandir el número de matrículas a cualquier precio, a cualquier costo, tuvo justamente el efecto contrario. La insuficiencia en el número de predios y profesores hizo que el tiempo necesario para la formación del alumno fuese reducido, posibilitando atender una "clientela mayor".

La verdad es que ya se hace difícil ocultar la descaracterización de nuestro movimiento educacional. Se puede expandir, por el simple aumento de participantes, un expectáculo, un acto recreativo, en 
rigor algo consumista, pero no se puede expandir, solamente por el aumento de participantes, un proceso, temporal y espacial, largo y complejo, de preparación individual, como es el educativo. Y lo que venimos haciendo es, en gran parte, la expansión del cuerpo de participantes, con el congestionamiento de la matrícula, la reducción del horario, la improvisación de escuelas de todo tipo, sin las mínimas condiciones necesarias de funcionamiento (Teixeira, 1994: 85).

Según Nosella (1997), tales mudanzas “oscurecieron el antiguo patrón unitario de calidad de la escuela", y contribuyeron al establecimiento de una escuela sin calidad. La continuidad del proceso de transformación de la escuela en universal, pública y gratuita, pasa a proporcionar apenas números de cupos. Una vez que es sabido que el índice de analfabetismo y el número de nińos en la escuela son indicadores sociales, cuya mala disposición provoca restricciones de cuño económico, como el control de organismos internacionales (ONU, Banco Mundial y FMI, por ejemplo), las políticas públicas dan énfasis casi exclusivo a la expansión de la educación.

Con la expansión desordenada de la enseñanza pública volcada para la población de baja renta, la educación privada adquiere gran significado, pues la clase más favorecida que hasta entonces hacía uso de la educación pública, que poseía una calidad incuestionable, pasa a utilizar la privada para garantizar la distinción educacional. De esta forma, el dualismo en nuestra sociedad permanece. Si antes era determinado por los que tenían acceso a la escuela y por los que no lo tenían, ahora es instaurado por la oposición de la educación pública versus privada.

Dentro de esta exposición acerca de la educación básica, es interesante asociar su trayectoria a hechos que han ocurrido en la educación superior. La democratización del acceso a la educación superior también ha sido realizado sin preocupación por la calidad y buscando resultados inmediatos. Las políticas nuevamente son asistencialistas e inmediatistas. Como ejemplo se puede citar el caso del Pro-Uni, programa estatal que incluye alumnos de baja renta en la educación superior privada, financiando sus estudios en facultades de credibilidad muchas veces dudosa, en lugar de invertir en el mejoramiento de la calidad de la educación básica para posibilitar el acceso a las universidades públicas, consideradas en general de mejor calidad.

En resumen, es necesario reconocer que la solución del problema de la mala calidad de la educación en el país pasa por una mejora de la calidad de la educación para la población más pobre. Como bien afirmó Nosella (1997: 180), eso "costará, sobre todo, la renuncia a ciertas ilusiones idealistas, pues es duro confesar que la educación escolar seria y de calidad, entre nosotros, está de hecho superconcentrada". 


\section{Educación y desigualdad de renta en el Brasil}

La desigualdad de renta en el Brasil es elevada desde el inicio de su colonización, estando ésta fuertemente concentrada en los dueños de los ingenios. Según Furtado (1979), los hombres de varios oficios y los supervisores del trabajo esclavo se apropiaban de menos de $2 \%$ de los rendimientos generados en el sector azucarero, en tanto que los pagos hechos a los ganaderos, leñadores y demás grupos de la población no pasaban el 3\% de la renta total. Para el autor, todo indica que por lo menos $90 \%$ de la renta de la colonia estaba concentrada en manos de los propietarios de ingenios de plantaciones de cańa.

La economía primaria-exportadora del Brasil colonial se desarrolló basada en el gran latifundio y en el trabajo esclavo, lo cual garantizó que la renta estuviese siempre muy concentrada en las manos de los grandes propietarios de la tierra. La agricultura de subsistencia sólo era aceptada por la Corona portuguesa como una actividad de apoyo al monocultivo. El sistema de capitanías hereditarias, caracterizadas por grandes extensiones de tierras cedidas por la Corona portuguesa, garantizó que casi toda la producción fuese volcada al mercado europeo, generando lucros y, consecuentemente, tributos a Portugal. Ese sistema estuvo vigente durante todo el periodo colonial y durante las primeras décadas del Imperio brasilero.

No obstante las transformaciones económicas, políticas y sociales ocurridas en el siglo XIX con la independencia del Brasil, el crecimiento de la caficultura, la inmigración europea y el fin de la esclavitud, la concentración de renta en nuestro país permaneció elevada, lo que en gran parte puede ser atribuido a la concentración de la propiedad de la tierra. Para Linhares y Silva (1999), el advenimiento de la República no significó una mudanza radical en el pasado agrario brasilero, lo que puede ser claramente observado con la instauración de la llamada "república de los coroneles". La política de indemnización a los negros libertos por los siglos de esclavitud, propuesta por Rodolfo Dantes y Rui Barbosa, ejemplo de uno de los ítems más avanzados de la pauta abolicionista, fue luego dejada de lado. De este modo, en el año de la Ley Áurea, existían más de 700 mil esclavos, pero nadie fue objeto de cualquier tipo de indemnización, mucho menos a través de un programa de reforma agraria. Junto con la libertad, los ex-esclavos cargaron consigo el peso de la miseria y el desinterés de las autoridades.

Barros et al (2000) llegan a considerar las desigualdades sociales, en particular la desigualdad de renta, como algo tan arraigado en la historia brasilera que adquiere estatuto de cosa natural. Según Ney (2002), a pesar de ser la desigualdad de renta un problema histórico, los primeros estudios sobre la distribución de renta en el Brasil, basados en datos representativos de la población, fueron realizados apenas a partir de la década de los ańos setenta. Comparando los 
resultados de los censos de las décadas de los años sesenta y de los setenta, se puede observar que la desigualdad de renta en los años sesenta no sólo era muy elevada, sino también que el acelerado proceso de industrialización, ocurrido en los primeros ańos del gobierno dictatorial, vino acompañado de un fuerte aumento de la desigualdad. El índice de Gini subió de 0,50 en 1960 a 0,57 en 1970, un aumento de 14\%. En este periodo el país se convirtió en campeón en el crecimiento de la desigualdad en una década (Fishlow, 1972; Langoni, 1973; Hoffmann, 2000; Ney, 2002).

El interés de reducir la desigualdad se debe también al hecho de que esta es una importante opción para la disminución de la pobreza, pues el Brasil puede ser considerado el país más rico entre los países con mayor número de miserables (Barros, et al. 2000). De acuerdo con el estudio de Barros, et al. (2000), a pesar del Brasil poseer un gran porcentaje de pobres, no puede ser considerado un país pobre, pues su población no está entre las más pobres del mundo. Tal afirmación está basada en una comparación internacional en cuanto a la renta per cápita, que coloca al Brasil entre el tercio más rico de los países del mundo, pues $64 \%$ de los países del planeta tienen renta per cápita menor que la del Brasil.

Así, comparando los países industrializados, el Brasil no es un país rico, pero, comparado a otros países en desarrollo estaría, en principio entre los que presentan mejores condiciones de enfrentar la pobreza de su población (Barros 2000: 26).

Según Barros et al. (2000: 27), en el Brasil la población pobre corresponde a aproximadamente el $30 \%$ de la población total, mientras que en otros países con renta per cápita semejante ese valor, en promedio, es menor que 10\%. En este sentido, "en el caso de que la desigualdad de renta en el Brasil correspondiese a la desigualdad mundial promedio asociada a cada desigualdad de renta per cápita, apenas $8 \%$ de la población brasilera debería ser pobre" (ver gráfico 1).

Esto evidencia el fuerte efecto que el grado de desigualdad en la distribución de renta en el Brasil tiene sobre la pobreza. Un estudio del IPEA (2005) muestra que, considerando la distribución de la renta domiciliar per cápita en el país, los $10 \%$ más ricos y $1 \%$ más ricos se apropian, respectivamente, de $46 \%$ y de $13 \%$ de la renta total. La concentración de renta es tan elevada que el $50 \%$ más pobre se apropia de apenas $13,3 \%$ de la renta total, proporción ligeramente superior a la del 1\% más rico. El índice de Gini que mide la desigualdad en la distribución es de 0,60 .

El nivel de pobreza, en cualquier país, tiene dos dimensiones: el nivel de renta y el nivel de desigualdad. Está claro que cuanto menor sea el nivel de renta de una región, determinada la desigualdad, mayor será la tendencia a la pobreza. 
No obstante, según Barros, et al. (2000) el hecho de que el país presente una de las mayores concentraciones de renta en el mundo no deja duda de que:

El extraordinario grado de desigualdad de renta brasilero se encuentra en el núcleo de la explicación de por qué el grado de pobreza en Brasil es significativamente más elevado que en otros países con renta per cápita similar (Barros, 2000: 34).

Como la desigualdad de renta es la gran responsable por la pobreza en el país, es importante analizar los efectos de sus principales causas. En la literatura, los principales determinantes de la desigualdad de rendimientos y que cuentan con estimativas sobre sus efectos son: la educación, los prejuicios de color y de género, las desigualdades entre regiones y sectores de actividades (agricultura, industria y servicios) y la distribución de riquezas. Entre ellos la educación es considerada el más fuerte (Fishlow, 1972; Langoni, 1973; Hoffmann, 2000; Ney, 2002).

Los autores frecuentemente sugieren la posibilidad de que el efecto de la educación en la desigualdad está sobrestimado, porque algunos modelos estadísticos no consideran importantes determinantes de la renta, tales como la dotación inicial de riqueza y el origen familiar, entre otras variables que no son investigadas por el Instituto Brasilero de Geografía y Estadística (IBGE), órgano responsable por las principales fuentes de datos de las investigaciones. No obstante conforme afirmó Ney (2006: 26)

A pesar que haya de hecho imperfecciones en las estimativas, la educación es sin duda uno de los principales determinantes de renta de los individuos, constituyendo un camino clásico de ascensión social. ${ }^{1}$ Independientemente de las divergencias sobre su papel en la reducción en la desigualdad, hay consenso en que la promoción de la educación básica en el país es condición necesaria para el ejercicio pleno de la ciudadanía y para la participación adecuada de las personas en una economía moderna. Además de esto, la educación es un determinante significativo del nivel de productividad y rendimiento del trabajo, y los resultados de los estudios publicados desde los años 70 presentan evidencias suficientes para que pueda ser destacada como uno de los importantes factores responsables por la conformación de la renta en el país.

Para Ney (2006), así las estimativas sobre los efectos de los factores determinantes de las disparidades de rendimiento carezcan de mayor precisión,

1 Hasta los autores que resaltan la posibilidad de que exista una exageración (sesgo) en la evaluación del efecto de la variable, como Fishlow (1973), Hoffmann (2000) y Ueda (2001), la consideran uno de los principales condicionantes de la concentración de renta en el país. Para Fishlow (1973: 45), por ejemplo a pesar de haber en la década de los setenta una sobrevaluación en el nivel de desigualdad explicado por la educación, esto no debería "significar el desprecio por la política educacional, un instrumento muy importante en cualquier estrategia de la disminución de la desigualdad de renta". 
el efecto de la educación en la desigualdad es seguramente fuerte. En este sentido, la reducción de las desigualdades educacionales en el país puede ser considerada una de las principales políticas a favor de la disminución de la concentración de renta.

\section{Desigualdad de oportunidad educacional}

Como fue dicho anteriormente, hasta los años treinta en la república vieja, donde el poder estaba concentrado en las manos de la oligarquía agraria, el acceso a la educación era privilegio de una minoría favorecida, dejando al pueblo el analfabetismo o, para algunos pocos, la educación primaria. Con el fin de la era Vargas, en el pequeño intervalo democrático se intensifican las luchas por la democratización del acceso a la educación, por una "escuela pública, universal, gratuita y laica" (Frigotto, 2005: 229).

Sin embargo, los veinte ańos de la dictadura militar detienen por la fuerza ese proceso y el campo educacional es modificado significativamente por dos reformas: la reforma universitaria de $1968^{2}$ y la reforma de la educación primaria y secundaria de $1971 .{ }^{3}$

Se trata de reformas que se dan bajo los auspicios de la Teoría del Capital Humano, o del economicismo de la educación. El efecto ideológico de estas reformas, sobre el ideario del capital humano es que se desplaza el problema de la desigualdad de clase social para la desigualdad educacional y con eso se pasa la idea de que la superación de aquella desigualdad se da por la educación. Al final de los veinte años de la dictadura, la desigualdad educacional continuó y la de clase se profundizó (Frigotto, 2005: 229).

Existe, según la literatura una relación directa entre la desigualdad de renta y la desigualdad de oportunidad educacional. ${ }^{4}$ Ferreira (2000) llega a sugerir la

2 "La reforma universitaria de 1968 tuvo inicio, efectivamente, en 1967, con la edición de decretos leyes volcados para las instituciones federales de enseńanza. La ley 5540/68 y el decreto ley 464/69 consolidaron la reforma y la ampliaron para todos los sistemas de enseńanza. La reforma de 1968 representa, sin duda, un considerable avance en la modernización de la educación superior brasilera, objetados los aspectos autocráticos, frutos del régimen entonces vigente, centralizador y dictatorial" (ver Frauches Celso da Costa, p. 4 en: La libre iniciativa y reforma universitaria brasilera).

3 “En 1971, con la ley 5692, ocurrió una verdadera revolución en el sistema de enseñanza. En ese periodo, en cuanto a la legislación, todavía se preservaba la anterior distinción elitista de la escuela secundaria, en cuanto a los educadores, intelectuales y administradores, con raras excepciones continuaban defendiendo la selectividad y la función propedéutica de ese tipo de enseñanza, las poblaciones urbanas, por la fuerza de sus reivindicaciones, dieron inicio al proceso de transformación de la realidad educacional en el país." (ver Sposito, p.12 Marilia Pontes, en: O Povo vá à Escola).

4 Es necesario llamar la atención para que no se tenga una visión de la educación solamente de carácter económico, apenas como factor de crecimiento económico, ya que se trata, también, de un derecho constitucional. Además se sabe que a medida que el grado de escolaridad aumenta se tiene ese reflejo en diversas situaciones, destacándose el crecimiento poblacional, la organización de la familia, y la participación política, entre otros. 
necesidad de un re-direccionamiento del foco de los economistas que estudian la redistribución de renta en el Brasil hacia los mercados e instituciones educacionales:

Si es la educación la que explica tanto de nuestra distribución de renta, qué será lo que explica nuestro proceso de producción de educación y acumulación de capital humano? (Ferreira, 2000: 144).

A pesar de que los adeptos de la Teoría del Capital Humano puedan estar exagerando en la defensa de las políticas educacionales como forma de distribuir renta, omitiendo determinantes importantes de la desigualdad, tales como la concentración de la propiedad de la tierra y las desigualdades regionales, la democratización del acceso a la educación de calidad puede desempeñar un papel importante en la desconcentración de la renta. Ney (2006), por ejemplo, afirma que la baja escolaridad de la población relativamente pobre, en el medio rural, puede comprometer el éxito de una política de reforma agraria, teniendo en cuenta que $35,7 \%$ de la población adulta que vive en el campo tiene menos de un año de estudio y $69,4 \%$ no completaron la antigua primaria ( $4^{\mathrm{a}}$ serie).

Parece clara la idea de que existe en el país no sólo el sentido de causalidad educación-rendimiento, como también su relación en sentido inverso: rentaeducación. En cuanto al primer sentido, podemos afirmar que la contribución de la educación para las disparidades de rendimientos depende del nivel de desigualdad educacional. Cuanto mayor sea la heterogeneidad de la fuerza de trabajo, mayor tiende a ser la desigualdad de renta (Barros, et al. 2002).

En relación al segundo sentido, el de la renta influenciando a la escolaridad, uno de los graves problemas del sistema educacional brasilero siempre ha sido lograr mantener a los alumnos en la escuela. Los datos del gráfico 2 muestran que el número de alumnos pobres que ingresan en el primer año de enseñanza fundamental es alto, comparado a los demás países en desarrollo. No obstante, en la medida en que aumenta el nivel de escolaridad, la evasión escolar es gradual, una vez que los individuos pobres necesitan trabajar para suplir sus necesidades y las de su familia. Esto contribuye a que la tasa de evasión escolar de Brasil sea alta si es comparada con la de los demás países (ver gráfico 2).

La deserción escolar no sólo se debe a la dificultad financiera para mantener un hijo estudiando, también se debe al bajo incentivo de los padres. El punto clave apuntado por Barros, et al. (2001) es la escolaridad de los padres, que constituiría el principal determinante del desempeño educacional, llegando a superar el efecto de la renta familiar per cápita. La explicación se da por el hecho de que los padres más escolarizados consideran la inversión en educación 
de suma importancia para el futuro de sus hijos y tienden a incentivarlos más a continuar estudiando.

De acuerdo con estimativas del autor, un año o más de escolaridad de los padres tiende a elevar la escolaridad de los hijos en torno a 0,3 ańos de estudio, lo que daría un efecto semejante al incremento de $\mathrm{R} \$ 340,00$ en la renta per cápita (Barros et al., 2001). Según estos mismos autores, cuanto más pobre es la familia menos tiende ella a invertir en educación y

como el grado de pobreza de un individuo es fuertemente determinado por su nivel educacional, esa naturaleza diferenciada de la subinversión en educación lleva a la transmisión intergeneracional de la pobreza. Los individuos nacidos en familias pobres hoy tienden a tener escolaridad inferior, y serán con mayor probabilidad, los pobres del mañana (Barros, et al. 2001: 6).

Los estudios económicos que analizan el efecto de la condición socioeconómica de las familias en la educación observan, como regla, el nivel de escolaridad de las personas. El problema es que, conforme pudo ser observado en la primera sección, la expansión de la oferta de la enseńanza pública brasilera vino acompañada de una caída en la calidad de la educación. El dualismo educación pública versus privada, que garantiza educación de calidad a los pocos que poseen condiciones financieras para pagar las escuelas particulares y deja a los demás una educación pública ${ }^{5}$ que tiene como principal problema la baja calidad, hace que una persona de baja renta concluya la educación media en condición inferior a las de renta más elevada. Considerar solamente el tiempo de escolaridad nos llevaría, por tanto, a encontrar resultados que subestimarían el nivel de desigualdad de oportunidad educacional en el país. En este sentido, en la próxima sección será realizado un estudio de caso de la región noroeste fluminense. En él analizaremos el desempeño de los participantes en el Examen Nacional de Enseñanza Media (ENEM), relacionando las notas obtenidas en la prueba objetiva con informaciones sobre las características socioeconómicas de los estudiantes.

\section{Desigualdad en la calidad de la educación básica en la región noroeste fluminense}

La región noroeste fluminense se localiza en la parte más septentrional del interior del Estado de Rio de Janeiro. Ocupa cerca del 12\% del territorio total del Estado, siendo compuesta por trece municipios: Aperibé, Bom Jesús, Do

\footnotetext{
5 Sin generalizar, es de conocimiento general la calidad de los establecimientos federales de educación en los que se refiere a la educación de nivel medio. Se sabe que con relación a la educación superior este dualismo público versus privado permanece, no obstante se invierte, guardando también las debidas excepciones.
} 
Itabapoana, Cambuci, Italba, Itacoara, Itaperuna, Laje Do Muriaé, Miracema, Natividade, Porciúncula, Santo Antônio de Pádua, São José de Ubá, Varre-Sai.

Es importante resaltar que el Noroeste Fluminense es la mesoregión más pobre de todo el Estado de Rio de Janeiro. De acuerdo con los datos del último censo demográfico, la renta media de las personas responsables por los domicilios era de R \$ 517,3, valor 24,3\% menor de la observada en la Baixada Fluminense, R \$ 683,2, menor que la del Centro Fluminense, R $\$ 624,5$, menos de la mitad de la región metropolitana, $\mathrm{R} \$ 1045,5,15,3 \%$ inferior a la del Norte Fluminense, $\mathrm{R} \$$ 610,4 , y 29,6\% inferior a la del Sur Fluminense, $R \$ 734,3$ (ver tabla 1). ${ }^{6}$

Tabla 1 - Nivel de escolaridad y valor del ingreso medio mensual de las personas con ingresos que son responsables por los domicilios. Noroeste fluminense, 2000.

\begin{tabular}{|l|c|c|c|c|c|c|c|}
\hline \multirow{2}{*}{ Estadistica } & \multicolumn{2}{|c|}{ Ingreso $(\mathbf{R}$ ) } & \multicolumn{5}{c|}{ \% con ańos de escolaridad } \\
\cline { 2 - 8 } & Medio & Mediano & $\begin{array}{c}\text { Inferior } \\
\text { a 1 año }\end{array}$ & $\begin{array}{c}\mathbf{1} \text { a 3 } \\
\text { años }\end{array}$ & $\begin{array}{c}\text { 4 a 7 } \\
\text { años }\end{array}$ & $\begin{array}{c}\mathbf{8} \text { a 10 } \\
\text { años }\end{array}$ & $\begin{array}{c}\mathbf{1 1} \text { ańos } \\
\text { o más }\end{array}$ \\
\hline Baixada & 683,2 & 380,0 & 12,6 & 20,4 & 33,2 & 13,3 & 20,6 \\
\hline $\begin{array}{l}\text { Centro } \\
\text { Fluminense }\end{array}$ & 624,5 & 310,0 & 13,2 & 19,9 & 34,8 & 13,1 & 18,9 \\
\hline Metropolitana & $1.045,5$ & 500,0 & 7,1 & 12,8 & 30,2 & 17,2 & 32,6 \\
\hline $\begin{array}{l}\text { Noroeste } \\
\text { Fluminense }\end{array}$ & 517,3 & 250,0 & 17,7 & 20,4 & 32,1 & 11,5 & 18,3 \\
\hline $\begin{array}{l}\text { Norte } \\
\text { Fluminense }\end{array}$ & 610,4 & 300,0 & 14,5 & 20,4 & 32,0 & 12,9 & 20,2 \\
\hline $\begin{array}{l}\text { Sur } \\
\text { Fluminense }\end{array}$ & 734,3 & 412,0 & 9,2 & 16,4 & 35,1 & 15,5 & 23,9 \\
\hline
\end{tabular}

Fuente: IBGE (2008).

Otro indicador importante para analizar las desigualdades inter-generacionales presentes en el Estado es el nivel de escolaridad de las personas. Considerando nuevamente a las personas responsables por los domicilios puede observarse que, en la región noroeste fluminense, $17,7 \%$ tiene escolaridad inferior a 1 año y $38,1 \%$ no concluyó la antigua primaria, que corresponde hoy al primer ciclo de enseñanza fundamental ( $4^{\mathrm{a}} \mathrm{grado} / 5^{\circ}$ año). Vale la pena resaltar que menos de 30,0\% de los jefes de los domicilios habían concluido la enseńanza fundamental. En la región metropolitana, por ejemplo, el número de personas 
con escolaridad inferior a un año cae a 7,1 \% y prácticamente la mitad de los jefes habían concluido la enseñanza fundamental.

Teniendo en consideración que las condiciones socioeconómicas de las familias, particularmente el nivel de renta y escolaridad de los padres, son considerados determinantes importantes del desempeño educacional de los hijos, se puede esperar que el desempeño de los participantes del ENEM en el Noroeste Fluminense sea el peor de todas las mesoregiones del Estado. En los próximos ítems veremos hasta qué punto esta hipótesis es verdadera.

\section{Objetivos y metodología}

A partir de los microdatos del Examen Nacional de Enseñanza Media (ENEM) de 2005, se pretende trazar un perfil de la calidad de la educación básica en la región Noroeste Fluminense, relacionando las notas obtenidas en el examen objetivo con informaciones obtenidas por el cuestionario socioeconómico respondido por los participantes del examen. Comparando los resultados de alumnos por renta familiar, escolaridad del padre y tipo de escuela (pública o privada), entre otros factores, se pretende analizar la heterogeneidad educacional en la región.

La importancia de la educación básica radica no sólo en la preparación para la participación social como ciudadano, sino también en la posibilidad que otorga de seguir los estudios de educación superior, que permiten posteriormente una mejor inclusión en el mercado de trabajo. A su vez, la desigualdad en la calidad de la educación básica adquirida por alumnos ricos y pobres en el sistema de enseńanza brasilero puede ser revelada por el desempeño en las pruebas del ENEM. Es importante analizar hasta qué punto estudiantes de escuelas públicas, hijos de padres pobres y con poco estudio, han presentado un desempeño peor que el de los estudiantes de padres ricos y más escolarizados.

El ENEM posee un examen objetivo con sesenta y tres preguntas de elección múltiple y un examen de redacción. Los alumnos son evaluados en los dos exámenes de forma independiente y reciben dos notas integrales distribuidas en una escala de 0 a 100 (MEC/INEP 2006 y Ney, et al. 2008). No obstante, como el objetivo de este trabajo es evaluar las diferencias en la calidad de educación recibida por alumnos ricos y pobres de la región Noroeste, nos limitamos a utilizar apenas la nota del examen objetivo, por dos razones: a) a pesar de existir un rígido sistema de corrección de la redacción, es imposible considerar la impersonalidad en la hora de determinar la nota, mientras que las pruebas objetivas poseen un sistema de corrección automatizado de lectura óptica de la tarjeta de respuestas, lo que neutraliza las opiniones de quien corrige la nota; $b$ ) 
el examen de redacción contempla un único tema, lo que permite la inclusión de un número menor de áreas de conocimiento. ${ }^{7}$

Con el objetivo de obtener informaciones sobre las condiciones económicas y profesionales de los alumnos y de sus familias, el ENEM aplica un cuestionario socioeconómico, el cual es distribuido a los estudiantes junto con el manual y la tarjeta de confirmación de la inscripción, siendo recogido en el día de realización del examen (MEC/EINEP 2006b). A pesar de que no sea obligatorio responder el cuestionario, más del $85 \%$ de los estudiantes del examen objetivo lo hicieron, lo que da un total de casi 130 mil alumnos (ver tabla 2).

Esta investigación utilizará la misma base de datos de Ney, et al. (2008), la cual está compuesta por los microdatos de la educación del ENEM 2005, excluyendo las personas que no hicieron el examen objetivo y no respondieron al cuestionario socioeconómico. El resultado es un total de 129.797 estudiantes, mas de tres cuartos provenientes de la región metropolitana y apenas 3\% de la región noroeste fluminense. Es importante señalar que más de 4.200 estudiantes hicieron el examen objetivo y respondieron el cuestionario, lo que nos permite tener un gran número de observaciones en todas las estimaciones.

Esta investigación se basa en una distribución de frecuencias. El número de aciertos es una variable discreta, es decir puede asumir un número finito de diferentes valores dentro de un intervalo finito que va de 0 a 63 preguntas. En este sentido, la distribución de frecuencias de las notas puede ser adecuadamente representada por gráficos de barra. Para construir una distribución de frecuencias se debe antes comenzar por la elección de los intervalos de clase, es decir por el intervalo de las notas. Los intervalos de notas escogidos serán los mismos sugeridos por el INEP, en los cuales el total de los aciertos obtenidos en las 63 preguntas, que poseen todas el mismo valor, es colocado en una escala que varia de 0 a 100 puntos. Después de colocado en la escala, los puntos (notas) son clasificados en tres líneas de desempeño: a) insuficiente a regular, que son las notas mayores o iguales a 0 y menores o iguales a 40 ; b) regular a bueno, que son las notas superiores a 40 o menores o iguales a 70 ; c) bueno a excelente, que son las notas superiores a 70 (ver tabla 3).

7 Según Ney (2008: 4), en el "caso del examen de redacción del ENEM 2005, en particular, los participantes tuvieron que construir un texto en prosa de tipo disertación argumentativa sobre el tema: "El trabajo infantil en la realidad brasilera”. Además de ser el examen de redacción sobre un único asunto, el tema propuesto está mucho más presente en la cotidianidad de los alumnos pobres, los cuales consecuentemente tendrían más "oportunidades" de aprender sobre el tema fuera del ambiente escolar y sin necesitar de libros, periódicos o revistas, al contrario que los alumnos ricos. Eso explica, en parte, el desempeńo substancialmente mejor de los participantes del ENEM, particularmente de los más pobres, en las pruebas de redacción que en las pruebas objetivas”. 
Tabla 3: Líneas de desempeño y número de aciertos.

\begin{tabular}{|c|c|}
\hline Líneas de desempeńo & Intervalos de notas \\
\hline Insuficiente a Regular & {$[0,40]$ - entre 0 y 25 aciertos } \\
\hline Regular a Bueno & $(40,70]$ - entre 26 y 44 aciertos \\
\hline Bueno a Excelente & $(70,100]$ - entre 45 y 63 aciertos \\
\hline
\end{tabular}

Fuente: MEC/INEP (2006a).

Según Ney, et al. (2008), no obstante ser las notas del examen del ENEM una buena referencia de la educación básica obtenida, pues la participación en el examen es voluntaria y está motivada por el deseo de ingresar en la educación superior, ellas presentan algunas limitaciones que deben ser consideradas en el análisis de los resultados obtenidos: a) Los resultados del ENEM pueden sobrestimar la calidad de la educación básica obtenida. Con el objetivo de ingresar a la universidad, los participantes del examen tienen la tendencia a dedicarse más al estudio en la educación media que los alumnos sin el mismo objetivo, lo que influenciará de forma positiva su proceso formativo. b) Hay siempre una oportunidad de responder bien una pregunta de elección múltiple por medio de una simple elección aleatoria. Como todas las preguntas poseen cinco alternativas posibles de ser marcadas, los estudiantes tienden a alcanzar un nivel medio de aciertos de un quinto de todas las preguntas respondidas por "adivinación".

A pesar de algunas limitaciones, Ney, et al. (2008) creen que los datos del ENEM 2005 ofrecen buena información para el análisis de la calidad de la educación básica recibida por los alumnos ricos y pobres. Los autores defienden la tesis de que las restricciones deben ser apuntadas para que sean tomados los debidos cuidados en el análisis de los resultados, considerando que la calidad de la educación básica debe estar sobreestimada.

\section{Resultados y discusión}

De acuerdo a lo que puede ser observado en el gráfico 3, además de la región Noroeste Fluminense presentar el menor nivel de escolaridad y renta en comparación con las demás mesoregiones fluminenses, también presenta la mayor proporción de personas con concepto insuficiente a regular (63,9\%) y menor porcentaje con concepto bueno a excelente en el ENEM $(2,1 \%)$ (ver gráfico 3).

Es común que alguien defienda que la mejor calidad de la enseńanza básica brasilera está en la red privada y que la calidad de la enseñanza nocturna y del 
supletivo $^{8}$ es significativamente inferior a la del diurno irregular. El gráfico 4 y la tabla 4 muestran, respectivamente, el desempeño de los participantes del ENEM según el tipo de escuela (pública o privada), la jornada (diurna o nocturna) y la modalidad de la educación (regular, profesional, o supletivo).

El análisis del gráfico 4 conduce a algunas consideraciones importantes. Primero, nos muestra la triste realidad de la escuela pública departamental y municipal, siendo evidente la disputa entre las mismas por la peor calidad, pues las diferencias entre ellas en todas las tres categorías de desempeño son poco expresivas. En la categoría insuficiente a regular, la red departamental obtuvo $72,8 \%$ contra $73,0 \%$ de la red municipal. Todavía nos sorprende el hecho de que ningún alumno de las escuelas municipales y apenas el $0,5 \%$ de los participantes provenientes de las escuelas departamentales obtuvieron notas superiores a 70 , o sea, cinco de cada mil alumnos de la red departamental tuvieron desempeño de bueno a excelente en el ENEM (ver gráfico 4).

El segundo aspecto a resaltar es la mejor calidad de los establecimientos federales de educación, que mostraron en sus porcentajes un aprovechamiento superior al de las escuelas particulares. En la red federal de enseñanza, la proporción de alumnos con concepto bueno a excelente es de 17,4\%, mientras que las instituciones particulares obtuvieron 15,6\%. Además de la buena calidad de la enseñanza, es preciso reconocer que parte de las diferencias existentes entre las escuelas públicas departamentales y federales se debe al hecho de que el ingreso a las instituciones federales se realiza a partir de un proceso selectivo, lo que acaba seleccionando a los buenos alumnos de la educación básica. De cualquier forma, sorprende el hecho de que la proporción de alumnos con notas superiores a 70 en las escuelas federales $(17,4 \%)$ es casi 35 veces mayor que en las escuelas departamentales $(0,5 \%)$.

Tabla 4: Distribución porcentual de los participantes del ENEM 2005, según la jornada y la modalidad que cursó o está cursando en la educación media y el concepto en el examen objetivo. Noroeste fluminense, 2005.

\begin{tabular}{|l|c|c|c|c|c|c|c|}
\hline \multirow{2}{*}{ Estadística } & \multicolumn{3}{|c|}{ Modalidad de la educación } & \multicolumn{3}{c|}{ Jornada de la educación media } \\
\cline { 2 - 7 } & Regular & $\begin{array}{c}\text { Técnico y } \\
\text { profesional }\end{array}$ & EJA1 & $\begin{array}{c}\text { Sólo } \\
\text { diurno }\end{array}$ & $\begin{array}{c}\text { Mayor } \\
\text { parte } \\
\text { diurno }\end{array}$ & $\begin{array}{c}\text { Mayor } \\
\text { parte } \\
\text { nocturno }\end{array}$ & Sólo \\
\hline $\begin{array}{l}\text { Insuficiente a } \\
\text { regular }\end{array}$ & 60,5 & 69,1 & 79,3 & 56,9 & 63,0 & 67,4 & 77,5 \\
\hline
\end{tabular}




\begin{tabular}{|l|c|c|c|c|c|c|c|}
\hline Bueno a regular & 37,2 & 29,9 & 20,4 & 40,2 & 34,3 & 32,4 & 22,3 \\
\hline $\begin{array}{l}\text { Bueno a } \\
\text { excelente }\end{array}$ & 2,3 & 1,0 & 0,3 & 2,9 & 2,7 & 0,3 & 0,3 \\
\hline Total & 100,0 & 100,0 & 100,0 & 100,0 & 100,0 & 100,0 & 100,0 \\
\hline
\end{tabular}

Fuente: Elaboración Propia.

Nota: Educación de Jóvenes y Adultos y supletivo.

La tabla 4, a su vez, muestra que el desempeńo de los alumnos de la Educación de Jóvenes y Adultos (EJA) y del supletivo, así como de quienes cursaron la enseñanza nocturna, fue significativamente inferior al de estudiantes de cursos regulares y diurnos. No obstante, queda bastante clara la mala calidad de la educación regular y diurna en la región, en la cual menos de 3\% de los estudiantes tuvieron desempeńo bueno a excelente y cerca de $60 \%$ tuvieron concepto de insuficiente a regular. Por lo tanto, el mejoramiento de la calidad de la educación básica en la región pasa necesariamente por la mejora de la educación en todos los turnos y modalidades de enseñanza.

Los gráficos 5 y 6 presentan, respectivamente, la distribución porcentual de los participantes del ENEM y relacionan los resultados obtenidos con el nivel de escolaridad del padre (gráfico 5) y con los estratos de renta familiar medida según el salario mínimo legal vigente (SMLV) (gráfico 6). El objetivo es medir directamente el efecto de la condición socioeconómica de la familia en la calidad de la educación básica adquirida, evidenciando los presupuestos teóricos presentados en los capítulos iniciales.

Como se puede observar en el gráfico 5 , a medida que la escolaridad paterna crece, es notable la reducción del porcentaje de participantes con concepto insuficiente a regular; al mismo tiempo ocurre un gran aumento en la proporción de personas con conceptos de regular a bueno y de bueno a excelente. Todavía es interesante observar que entre las categorías del padre con educación media incompleta y media completo, los cambios en las proporciones de personas tanto con concepto insuficiente a regular como con concepto regular a bueno son notorias. Lo mismo sucede en las categorías de personas con padres con educación superior incompleta y superior completa. (ver gráficos $\mathbf{5}$ y $\mathbf{6}$ )

Entre los individuos con padres sin estudio, más del 80,0\% obtuvieron una nota menor o igual a 40 y nadie sacó más de 70 puntos. La desigualdad de oportunidad educacional se hace evidente cuando observamos que $13,2 \%$ de los individuos con padres que concluyeron la educación superior sacaron más de 70 y menos de $30,0 \%$ se quedaron con una nota menor o igual a 40 .

El gráfico 6 muestra, una fuerte correlación positiva entre la renta familiar y el desempeño en el examen objetivo, ya que a medida que la renta aumenta 
la proporción de participantes del ENEM con concepto insuficiente a regular se reduce, al paso que la de las personas con concepto regular a bueno y de bueno a excelente aumenta. Un hecho que debe ser tenido en cuenta en este gráfico es la existencia en la categoría "ninguna renta" de $2,7 \%$ de personas con un resultado de excelente a bueno, quebrando la relación positiva entre desempeño y renta familiar. El motivo puede ser que algunos participantes con renta familiar más elevada no quisieron informar sobre el ingreso familiar y optaron por declarar renta nula en lugar de dejar la pregunta sin responder.

En este sentido, la condición socioeconómica de la familia, representada por la escolaridad de los padres y por la renta familiar, tiene un fuerte efecto en la calidad de la educación básica, lo que muestra la existencia de una enorme desigualdad en la calidad de la educación adquirida por alumnos ricos y pobres en la región. Alumnos venidos de escuelas estatales y los que cursaron la educación media durante la noche, tuvieron desempeño mediocre y significativamente inferior al de las personas que pudieron hacer un curso regular durante el día.

\section{Consideraciones finales}

En esta investigación hemos tenido la oportunidad de analizar el proceso de expansión de la escuela pública, la democratización del acceso a la educación y los conflictos durante ese recorrido. Entre las principales contradicciones cabe destacar el dualismo escolar. En un primer momento, antes del crecimiento del número de cupos en la red pública de enseñanza, éste simplemente excluía buena parte de la población pobre de las escuelas, y posteriormente determinó la calidad de la educación que cada individuo recibiría.

Las altas desigualdades de renta y de oportunidad educacional son dos problemas históricos. No obstante todas los cambios políticos, sociales y económicos ocurridos desde la independencia del país, ellas todavía representan dos grandes problemas sociales brasileros. El carácter desigual de nuestra sociedad es de hecho algo tan antiguo que acaba siendo considerado "natural". Y el hecho de que Brasil sea uno de los países con las mayores desigualdades de renta del mundo ocurre, en gran parte, debido a dos características extremas de nuestro sistema educacional: una elite altamente escolarizada y una población pobre sin condiciones de competir en el mercado de trabajo por obtener su base educacional en una escuela pública de baja calidad.

El análisis de la calidad de la educación básica en el noroeste del Estado de Rio de Janeiro, basada en los microdatos del ENEM, retrata bien esa realidad. La diferencia entre la calidad de la educación pública y privada en la región ocurre en relación a las escuelas municipales y departamentales. Por otro lado, las escuelas 
federales alcanzaron mejores desempeńos. Considerando el total de participantes del ENEM que estudiaron en escuelas departamentales y municipales, cerca del $0,5 \%$ obtuvo nota superior a 70 . En contrapartida, los alumnos de la escuela federal consiguieron las mejores notas. Con una proporción de 17,4\% del total de sus participantes en la categoría de desempeño bueno a excelente, esta institución consiguió superar los 15,6\% conquistados por los alumnos de la red privada.

La influencia de la escolaridad de los padres en el desempeńo educacional de los hijos, defendida por Barros, et al. (2001), se comprueba en la región Noroeste Fluminense, donde es evidente el mejor desempeño de los hijos de padres más escolarizados. La fuerte correlación positiva entre la nota en el examen objetivo y la renta familiar también evidencia la gran desigualdad en la calidad de la educación existente entre ricos y pobres en la región.

En este sentido, los datos dejan bastante clara la gran necesidad de que el mejoramiento de la educación pública sea objeto de políticas públicas, para que existan condiciones menos desiguales entre individuos en el acceso a la educación de buena calidad. Por restringir el ejercicio de ciudadanía y limitar el acceso de la población más pobre al mercado de trabajo, la educación es uno de los principales factores determinantes de la enorme desigualdad de renta en el Brasil.

\section{Anexo}

\section{Gráfico 1 - Relación entre nivel de pobreza y renta per cápita para un conjunto seleccionado de países.}

$\%$ de pobres (PO)

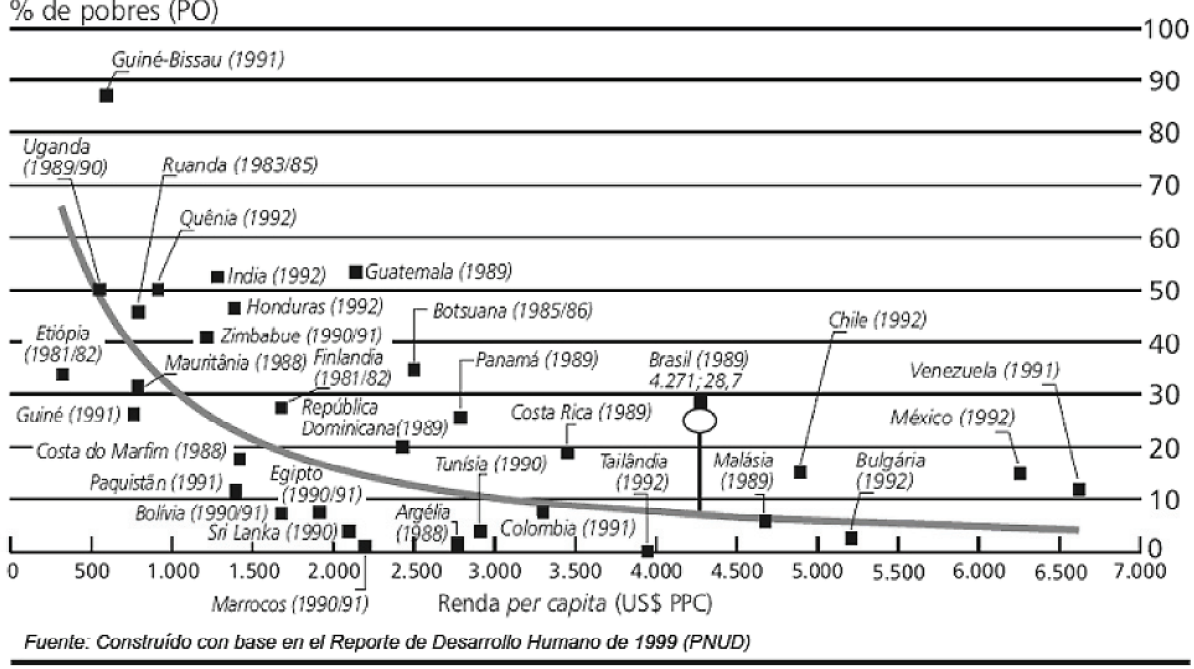

Fuente: Barros, et al. (2001). 
Gráfico 2- Porcentaje de los jóvenes pobres (de 15 a 19 años) que terminaron el primer ańo y porcentaje que terminaron el quinto ańo entre aquellos que terminaron el primero.

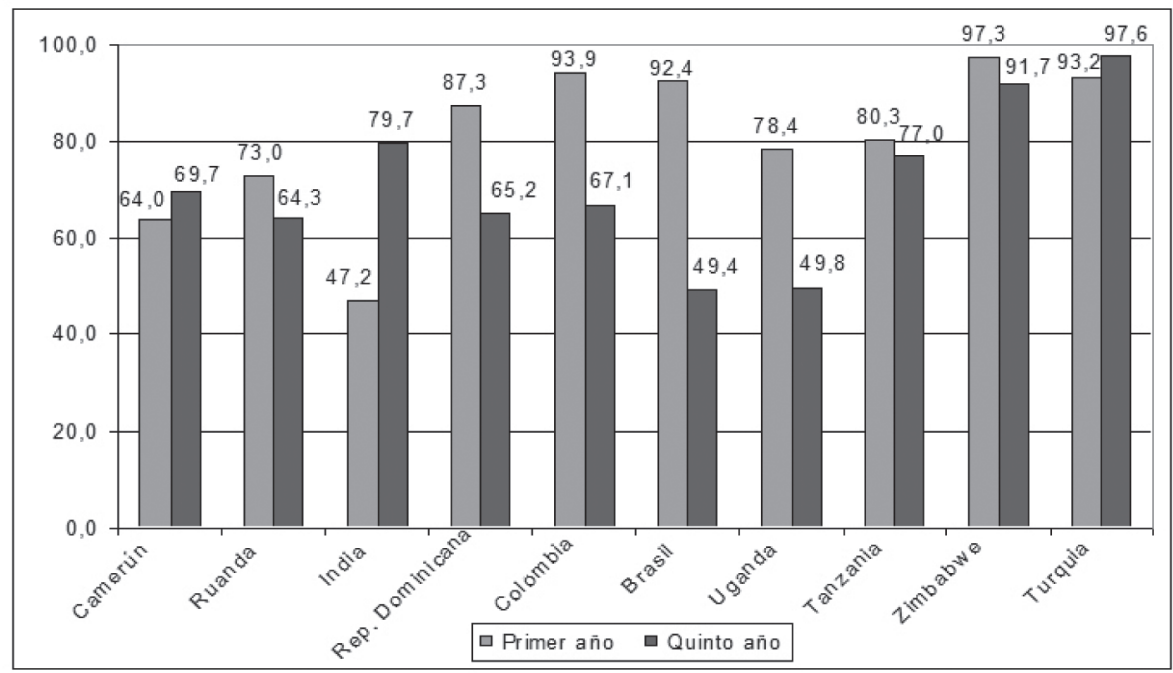

Fuente: Filmer e Pritchett (1998) apud Ney (2002)

Nota: Los jóvenes pobres son aquellos que están entre los $40 \%$ más pobres de la población.

Gráfico 3 - Desempeño de los participantes del ENEM, conforme mesoregiones. Río de Janeiro, 2005.

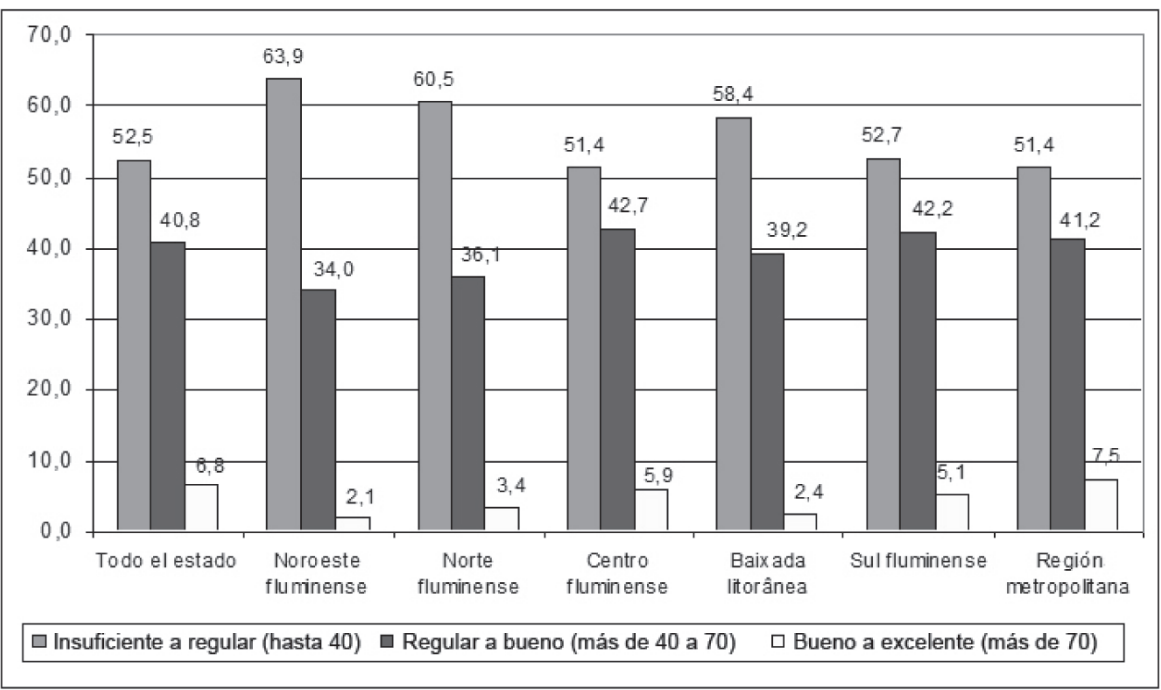

Fuente: Ney, et al. (2002) 
Gráfico4.-Distribución porcentual delos participantes delENEM2005, segúndependencia administrativa de la escuela y concepto en examen objetivo. Noroeste Fluminense, 2005.

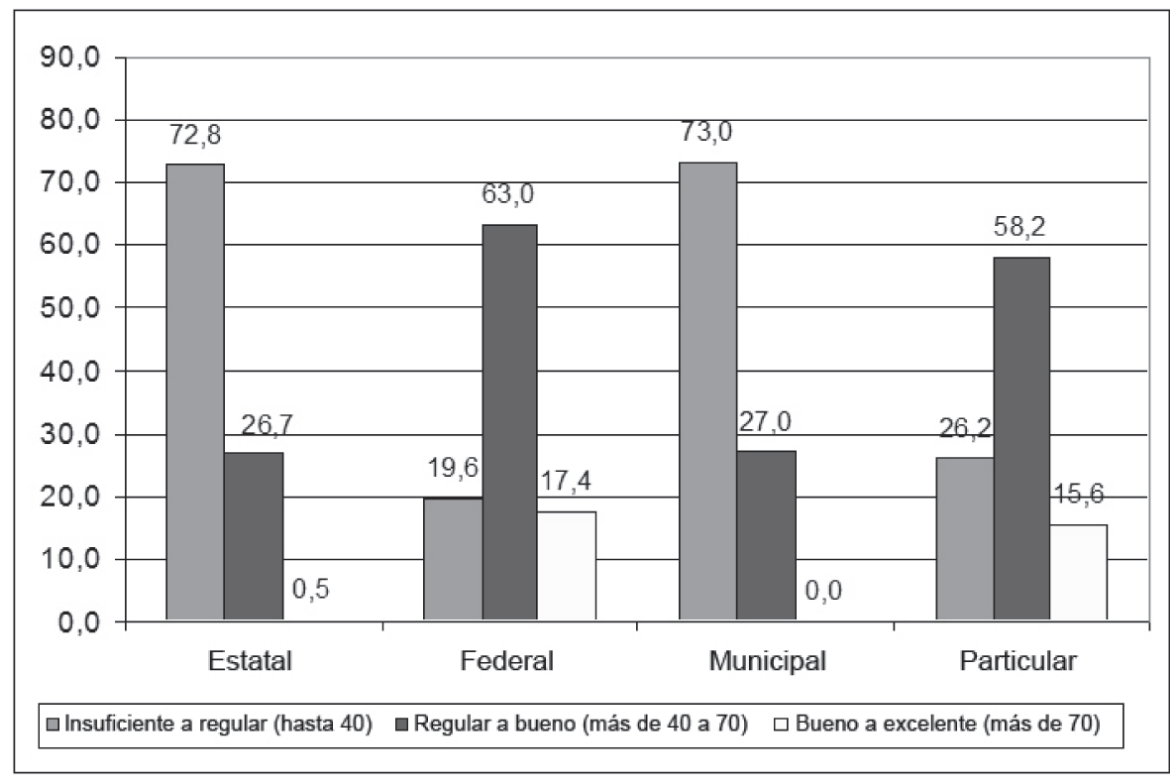

Fuente: Elaboración propia.

Gráfico 5: Distribución porcentual de los participantes del ENEM 2005, según el nivel de escolaridad del padre y concepto en el examen objetivo. Noroeste Fluminense, 2005.

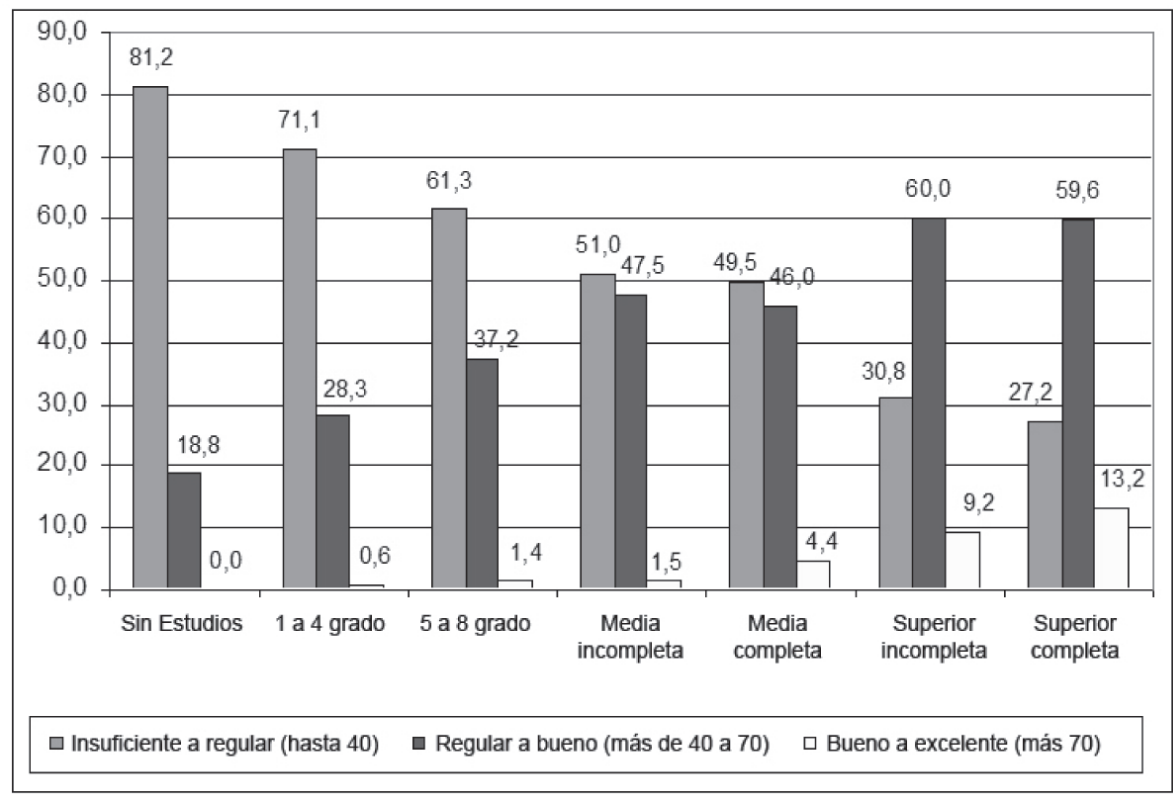

Fuente: Elaboración propia. 
Gráfico 6 - Distribución porcentual de los participantes del ENEM 2005, según el nivel de renta familiar en salarios mínimos (SM) y concepto en el examen objetivo. Noroeste Fluminense, 2005.

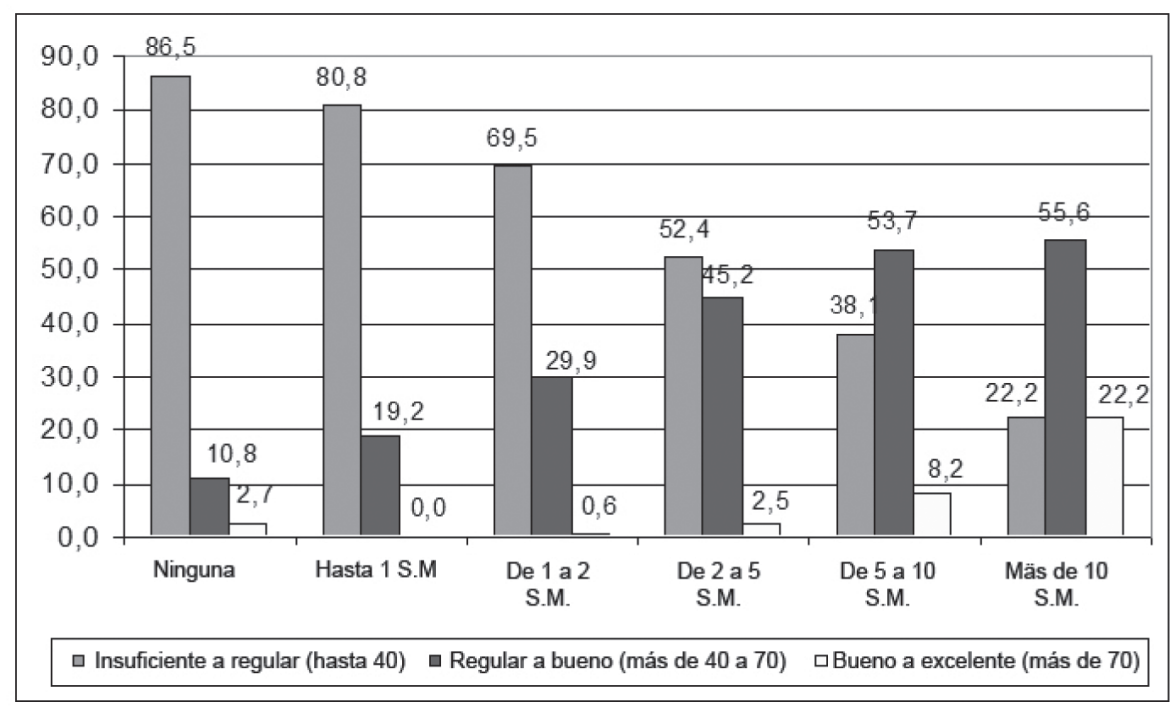

Fuente: Elaboración propia.

\section{Bibliografía}

-Barros, R. P., Henriques, R. E., Mendonça, R., (2000) "A estabilidade inaceitável: desigualdade e pobreza no Brasil”, en: Henriques, Ricardo (org.), Desigualdade e pobreza no Brasil, Rio de Janeiro, IPEA.

, (2002), "Pelo fim das décadas perdidas: educação e desenvolvimento sustentado no Brasil”, Rio de Janeiro, IPEA, texto para discussão no 857, 2002.

-Barros, R. P., Mendonça, R., Santos, D. D., y Quintaes, G., (2001), "Determinantes do desempenho educacional no Brasil", Rio de Janeiro, IPEA, texto para discussão n ${ }^{\circ} 834$.

-Ferreira, F. H. G., (2000) "Os determinantes da desigualdade de renda no Brasil: luta de classes ou heterogeneidade educacional?", en: Henriques, Ricardo (org), Desigualdade e pobreza no Brasil, Rio de Janeiro, IPEA. 
-Fishlow, A., (1972) "Brazilian Size Distribution of Income", en: The American Economic Review, VLXVII (2): 391-402, may, 1972, 2000.

-Furtado, C., (1979), Formação Econômica do Brasil, São Paulo, Nacional, 16a edição.

-Frigotto, G., (2005), "Escola pública brasileira na atualidade: liçóes da história”, enn: A escola Pública no Brasil História e Historiografia. Campinas, Autores Associados.

-Henriques, R., (2000), "Desnaturalizar a desigualdade e erradicar a pobreza: por um novo acordo social no Brasil”, en: Henriques, Ricardo (org), Desigualdade e pobreza no Brasil, Rio de Janeiro, IPEA, p.1-18, 2000.

-Hoffman, R., (2000), "Mensuraçấo da desigualdade e da pobreza no Brasil", en: Henriques, Ricardo (org), Desigualdade e pobreza no Brasil, Rio de Janeiro, IPEA, p. 82-107, 2000.

-Instituto Brasileiro de Geografia e Estatística, (2003), Censo Demográfico 2000 Educação - Resultados da amostra, Rio de Janeiro, IBGE.

-Instituto de Pesquisa Econômica Aplicada, Radar Social, Brasília, IPEA, 2005.

-Langoni, C. G., (1973), Distribución da renta e desenvolvimento econômico do Brasil, Rio de Janeiro, Expressão e Cultura.

-Linhare, M. Y. y Silva, F. C. T., (1999), Terra Prometida: Uma História da Questão Agrária no Brasil, Rio de Janeiro, Campus.

-Luzuriaga, L., (1959), História da educação pública, São Paulo, Nacional.

-Manacorda, M. A. A, (1996), "Educação no quinhentos e no seiscentos", en: História da educação da antiguidade aos nossos dias, São Paulo, Cortez, 5a edição. , (1996), "A educação no setecentos", en: História da educação da antiguidade aos nossos dias. São Paulo, Cortez. $5^{a}$ edição. 
-MEC/INEP/DEAES, (2006), Sinopses estatísticas da educação superior, Brasília, MEC/INEP.

-MEC/INEP, (2004), Relatório final Enem 2003, Brasília, MEC/INEP. , (2006a), Resultados do ENEM 2005: análise do perfil socioeconômico e do desempenho dos participantes, Brasília, MEC/INEP. , (2006b), Microdados do ENEM 2005, Brasília, MEC/INEP. , (2006c), Sinopse estatística: censo escolar da educação básica. Brasília, MEC/INEP.

-Ney, M. G., (2002), "Equação de rendimentos: O efeito da posse da terra", Dissertação de mestrado en Desenvolvimento Econômico apresentada ao Instituto de Economia da UNICAMP, Campinas, SP.

, (2006), Educação e desigualdade de renda no meio rural brasileiro, Tese de doutorado en Economia Aplicada apresentada ao Instituto de Economia da UNICAMP, Campinas, SP.

-Ney, M. G., Carvalho, A. M., y Reid, (2008), A influência das condiçóes socioeconômicas das famílias no desempenho dos participantes do ENEM na regiâo Norte Fluminense, Artigo ainda náo publicado. Campos dos Goytacazes, UENF.

-Nosella, P. A., A, (1997), "Escola Brasileira no final de um século: um balanço", en: Frigotto, G., Educaçâo e a crise do trabalho: perspectivas de final de século, Petrópolis: Vozes, 1997.

-Romanelli, O, (2005), História da educação no Brasil, Petrópolis, Vozes.

-Saviani, D. (1997), "Introdução", en: A nova Lei da Educação: Trajetórias, Limites e Perspectivas. Campinas, Autores Associados.

, (2005), "História da Educação Pública no Brasil: questões para pesquisa”, en: A escola Pública no Brasil História e Historiografia, Campinas, Autores Associados. 
-Smith, A. (1983), A riqueza das naçôes: investigação sobre sua natureza e suds causas, São Paulo, Abril Cultural.

-Teixeira, A., (1994), Educaçâo não é privilégio, Rio de Janeiro, UFRJ. , (1996), Educação é um direito, 2a edição. Rio de Janeiro, UFRJ.

-Ueda, M. E. (2001), "Educação e rendimentos: uma abordagem econométrica", Dissertação de mestrado apresentada ao Instituto de Economia da UNICAMP, Campinas.

-Veiga,, I. P. (2001), Projeto político-pedagógico da escola: uma construção possivel, Campinas, Papirus, $13^{a}$ edição. 